Article

\title{
Non-Resonant Magnetoelectric Energy Harvesting Utilizing Phase Transformation in Relaxor Ferroelectric Single Crystals
}

\author{
Peter Finkel ${ }^{1, *}$, Richard Pérez Moyet ${ }^{2, \dagger}$, Marilyn Wun-Fogle ${ }^{3, \dagger}$, James Restorff ${ }^{3, \dagger}$, \\ Jesse Kosior ${ }^{4,+}$, Margo Staruch ${ }^{1,+}$, Joseph Stace ${ }^{5,+}$ and Ahmed Amin ${ }^{5,+}$
}

Received: 11 November 2015; Accepted: 25 December 2015; Published: 30 December 2015

Academic Editor: Kenji Uchino

1 Materials Science and Technology Division, Naval Research Laboratory, Washington, DC 20375, USA; margo.staruch@nrl.navy.mil

2 Department of Materials Science and Engineering and Institute of Materials Science, University of Connecticut, Storrs, CT 06269, USA; richard.perez_moyet@uconn.edu

3 Survivability, Structure and Materials Department, Naval Surface Warfare Center, Bethesda, Carderock Division, Bethesda, MD 20817, USA; marilyn.wun-fogle@navy.mil (M.W.-F.); james.restorff@navy.mil (J.R.)

4 Department of Physics and Astronomy, Rowan University, Glassboro, NJ 08028, USA; kosior20@students.rowan.edu

5 Sensors and Sonar Systems Department, Naval Undersea Warfare Center Newport, Newport, RI 02841, USA; joseph.stace@navy.mil (J.S.); ahmed.amin@navy.mil (A.A.)

* Correspondence: peter.finkel@nrl.navy.mil; Tel.: +1-202-767-4481; Fax: +1-202-767-440-11

+ These authors contributed equally to this work.

\begin{abstract}
Recent advances in phase transition transduction enabled the design of a non-resonant broadband mechanical energy harvester that is capable of delivering an energy density per cycle up to two orders of magnitude larger than resonant cantilever piezoelectric type generators. This was achieved in a [011] oriented and poled domain engineered relaxor ferroelectric single crystal, mechanically biased to a state just below the ferroelectric rhombohedral $\left(\mathrm{F}_{\mathrm{R}}\right)$-ferroelectric orthorhombic $\left(\mathrm{F}_{\mathrm{O}}\right)$ phase transformation. Therefore, a small variation in an input parameter, e.g., electrical, mechanical, or thermal will generate a large output due to the significant polarization change associated with the transition. This idea was extended in the present work to design a non-resonant, multi-domain magnetoelectric composite hybrid harvester comprised of highly magnetostrictive alloy, [ $\mathrm{Fe}_{81.4} \mathrm{Ga}_{18.6}$ (Galfenol) or $\mathrm{Tb}_{x} \mathrm{Dy}_{1-x} \mathrm{Fe}_{2}$ (Terfenol-D)], and lead indium niobate-lead magnesium niobate-lead titanate (PIN-PMN-PT) domain engineered relaxor ferroelectric single crystal. A small magnetic field applied to the coupled device causes the magnetostrictive element to expand, and the resulting stress forces the phase change in the relaxor ferroelectric single crystal. We have demonstrated high energy conversion in this magnetoelectric device by triggering the $\mathrm{F}_{\mathrm{R}}-\mathrm{F}_{\mathrm{O}}$ transition in the single crystal by a small ac magnetic field in a broad frequency range that is important for multi-domain hybrid energy harvesting devices.
\end{abstract}

Keywords: energy harvesting; magnetoelectric; inter-ferroelectric phase transformation; broadband; low frequency; multi-domain

\section{Introduction}

The need for a sustainable power source for micro devices, autonomous underwater vehicles, drones, robotics, and remote sensor networks has placed an increasing emphasis on ambient energy harvesting devices that exhibit high converted energy density per cycle with improved efficiency [1-6]. Ambient energy may be one or more of the following sources: mechanical vibration, 
thermal fluctuations, or electromagnetic radiation. A multi-domain energy harvester is a harvester capable of generating a usable output electrical power in response to variations in ambient conditions. It is desirable if such a harvester could respond not only to low level inputs, but also to low frequency excitations. For instance, traditional mechanical energy harvesters are based on resonant cantilever designs utilizing the linear piezoelectric mode. However, as the most effective power conversion only occurs at the characteristic resonant frequency of the harvester, they are narrow band. Therefore, to harness broadband mechanical vibrations, a multimodal approach with multiple harvesters must be used. Another solution to broaden the bandwidth of the harvesters and maximize energy output is complex mechanical frequency up-conversion techniques, allowing for tuning the resonant frequency of the harvester to match the resonance of the excitation source frequency [4].

However, we recently have demonstrated a giant electromechanical energy conversion in a non-resonant device capable of delivering an energy density per cycle nearly two orders of magnitude larger than traditional cantilever piezoelectric devices working in resonance mode [7]. Moreover, the device is capable of low frequency energy conversion in the Hertz region and is not frequency-limited up to several $\mathrm{kHz}$ by the piezoelectric crystal-load impedance [8]. This device is also capable of converting variations in environmental temperature to a usable electrical output [9] and therefore can be considered a dual-domain harvester. It operates on a phase transition transduction principle in relaxor ferroelectric crystals that was first reported by Finkel, Benjamin, and Amin [10] and was later utilized in a miniaturized class IV (flex-tensional) acoustic source [11].

This electro-mechanical energy conversion was manifested during a ferroelectric-ferroelectric phase transformation in [011] cut domain engineered perovskite lead titanate-based relaxor ferroelectric single crystals with compositions near the morphotropic phase boundary (MPB) [7-9]. It was found that under mechanical pre-stress, a relatively small oscillatory stress or electric field drives the material reversibly between the ferroelectric rhombohedral $\left(\mathrm{F}_{R}\right)$ and ferroelectric orthorhombic $\left(\mathrm{F}_{\mathrm{O}}\right)$ states with a remarkably high polarization and strain jump induced at room temperature. This inter-ferroelectric phase transformation takes place via polarization rotation from the three-fold symmetry axes of the two rhombohedral macro-domain states to the two-fold symmetry axis of the mono-domain orthorhombic state. It is a consequence of the ultra-low energy barrier to stress- or electric field-induced inter-ferroelectric phase transformation due to the near degeneracy of the energy surface for lead indium niobate-lead magnesium niobate-lead titanate (PIN-PMN-PT) with a composition near the MPB [12]. Since the mono-domain $F_{O}$ state is unstable under free electric or elastic boundaries, it reverts back to its original $F_{R}$ state upon the removal of stress or field. In this work, we have employed the same concept to design and build a non-resonant, magnetoelectric (ME) composite harvester. Magnetostrictively driven strain in this harvester generates sufficient critical stress to induce this phase transformation in mechanically biased single crystal with demonstrated high electromechanical energy conversion in broad range of frequencies [7].

\section{Magneto-Electric Harvester Architecture and Characterization}

The ME hybrid harvester is comprised of a highly magnetostrictive $\mathrm{Fe}_{81.4} \mathrm{Ga}_{18.6}$ (Galfenol, from Etrema Products, Inc.) or $\mathrm{Tb}_{\mathrm{x}} \mathrm{Dy}_{1-x} \mathrm{Fe}_{2}$ (Terfenol-D) alloy and $\mathrm{Pb}\left(\mathrm{In}_{1 / 2} \mathrm{Nb}_{1 / 2}\right) \mathrm{O}_{3}-\mathrm{Pb}\left(\mathrm{Mg}_{1 / 3} \mathrm{Nb}_{2 / 3}\right) \mathrm{O}_{3}-$ $\mathrm{PbTiO}_{3}$ (PIN-PMN-PT) domain engineered relaxor ferroelectric single crystal under mechanical pre-stress. Commercially grown Bridgman PIN-PMN-PT single crystal with a nominal composition on the rhombohedral side of the MPB was selected to construct a magnetoelectric ME harvester. To determine the critical pre-stress, room temperature, quasi-static, harmonic compression-decompression scans of PMN-PMN-PT $4 \times 4 \times 12 \mathrm{~mm}^{3}$ bars cut in the 32-mode geometry and poled along the [011] direction were conducted using $0.1 \mathrm{~Hz}$ cycles with a $50 \mathrm{MPa}$ stress amplitude at different dc electric field levels from 0 to $0.5 \mathrm{MV} / \mathrm{m}$. Figure 1a depicts the stress-strain response of the crystal for different dc bias levels. A sharp strain change associated with a $\mathrm{F}_{\mathrm{R}}-\mathrm{F}_{\mathrm{O}}$ phase transformation occurs for the zero field response near a critical stress of $\sim 20 \mathrm{MPa}$ on compression. Note the magnitude of the critical 
stress decreases upon increasing the dc bias level as shown in Figure $1 \mathrm{~b}$ for both the compression and decompression cycles.
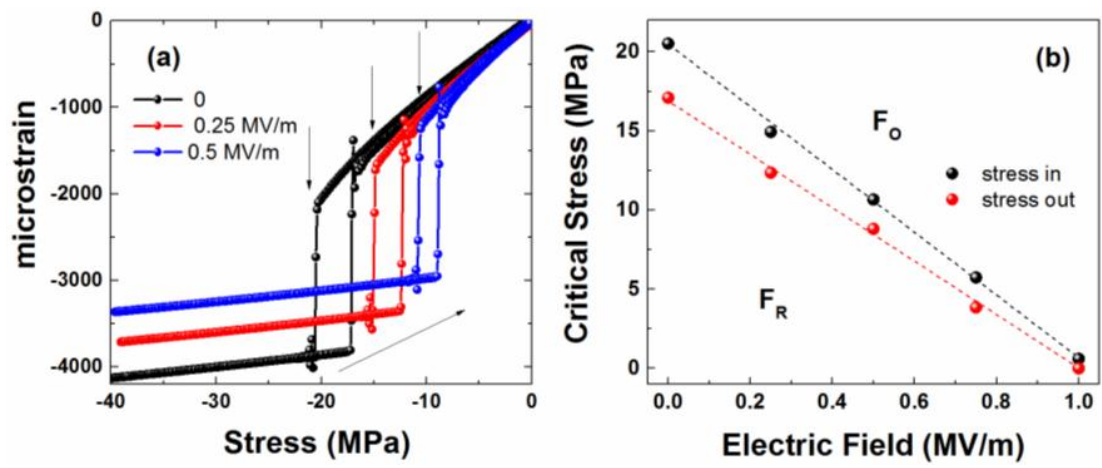

Figure 1. (a) Room temperature elastic response at various electric field bias and (b) stress-field stability region for [011] poled $\mathrm{Pb}\left(\mathrm{In}_{1 / 2} \mathrm{Nb}_{1 / 2}\right) \mathrm{O}_{3}-\mathrm{Pb}\left(\mathrm{Mg}_{1 / 3} \mathrm{Nb}_{2 / 3}\right) \mathrm{O}_{3}-\mathrm{PbTiO}_{3}$ (PIN-PMN-PT) samples, where $F_{R}$ and $F_{O}$ represent stable ferroelectric rhombohedral and ferroelectric orthorhombic states, respectively.

The hybrid system is designed to provide sufficient uniaxial stress in a pre-stressed piezocrystal with two specially machined stress-annealed Galfenol rods (with saturation magnetostriction $\sim 300 \times 10^{-6}$ ) encapsulated with the crystal inside the aluminum case (Figure 2a). The magnetostrictive Galfenol element's frequency range is primarily limited by eddy currents. These Galfenol rods have been laminated to reduce eddy current losses and have been used to demonstrate operation in $\mathrm{kHz}$ range (private communication J.R. and Etrema Products, Inc).

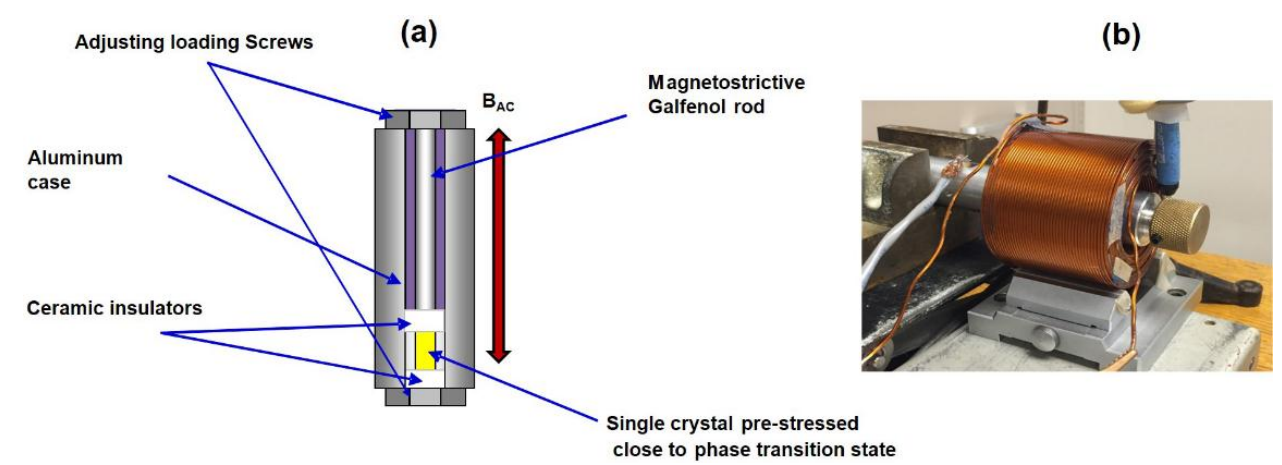

Figure 2. (a) Magnetoelectric harvester cross-section, showing the applied bipolar field direction.

(b) Experimental setup showing the harvester in a solenoid coil.

When driven by a varying magnetic field, this hybrid ME device utilizes the strain in the laminated Galfenol rod to further stress the PIN-PMN-PT crystal, triggering the $\mathrm{F}_{\mathrm{R}}-\mathrm{F}_{\mathrm{O}}$ phase transformation. The ME hybrid system is shown in Figure $2 a$, and the experimental setup in Figure $2 b$. Two polished ceramic (Macor@) spacers with insulating polyamide (Kapton) tape were used on both sides of the piezocrystal to avoid arc effects. To provide uniform and constant stress conditions for the PIN-PMN-PT, an elastomer (Polyurethane) spring was used for mechanically pre-stressing the piezocrystal close to a stress-induced $\mathrm{F}_{\mathrm{R}}-\mathrm{F}_{\mathrm{O}}$ phase transformation $(\sim 17 \mathrm{MPa})$ using the bottom pre-loading screw (Figure 2a). The end of one Galfenol rod is brought in intimate contact with the piezocrystal using an adjustable loading (top) screw allowing for the elimination of backlash to provide an additional magneto-mechanical stress. In this Galfenol rod, the stress annealing builds in about $30 \mathrm{MPa}$ of internal compressive stress which is added to the applied pre-stress reducing the requirement for an externally applied pre-stress $[13,14]$. This additional magnetostriction-driven 
stress variation resulting from an applied magnetic field is sufficient to trigger the $F_{R}-F_{O}$ phase transformation and consequent generation of voltage across [011] electrodes of the single crystal that is initially mechanically pre-stressed inside the aluminum case. Therefore, a direct magnetoelectric effect is realized here for direct energy conversion from a small ac magnetic field.

Figure 3a shows the magnetostrictive strain $(\lambda) v$ s. magnetic field $(H)$ curve and the piezomagnetic coefficient, $\mathrm{d} \lambda / \mathrm{d} H$, for a stress annealed Galfenol rod with $20.7 \mathrm{MPa}$ of pre-stress applied. The material is typically magnetically biased to operate in a quasi-linear regime of the device, with the vertical line representing an optimal magnetic bias point. This should correspond to the peak of the $d \lambda / d H$ of Galfenol as shown in Figure 3a at 200 Oe.
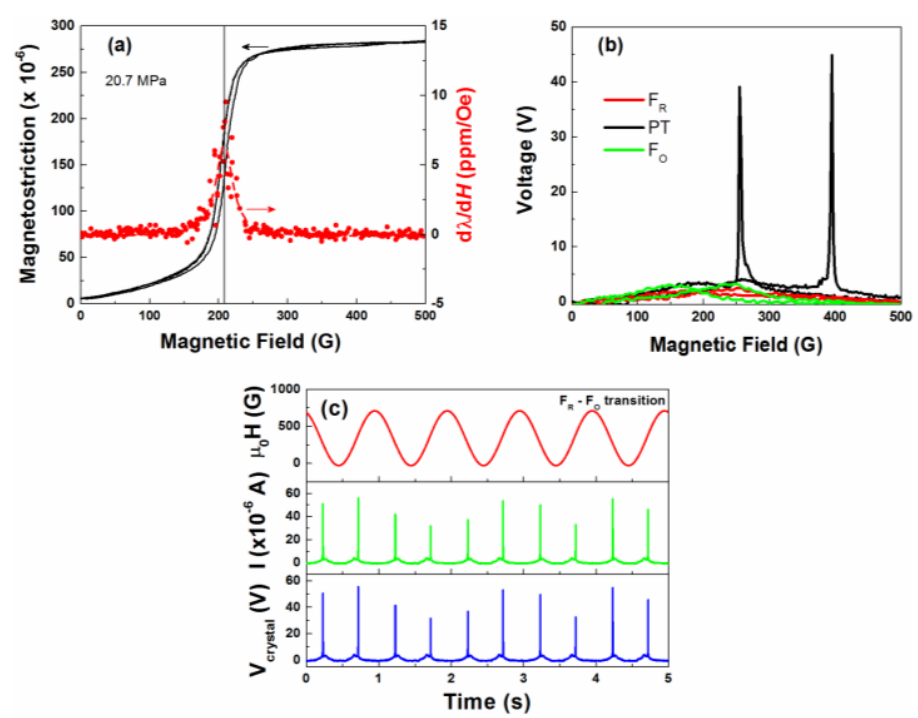

Figure 3. (a) Strain $v$ s. applied magnetic field and $\mathrm{d} \lambda / \mathrm{d} H$ for a stress-annealed Galfenol $\left(\mathrm{Fe}_{81.6} \mathrm{Ga}_{18.4}\right)$ rod with $20.7 \mathrm{MPa}$ of compressive pre-stress applied. The vertical line denotes a typical magnetic bias point. (b) Voltage generated by the piezocrystal under the three different pre-stress conditions described in the text. (c) Time dependence of the applied magnetic field, induced current and voltage from the crystal $\left(\mathrm{V}_{\text {crystal }}\right)$ in the PT region while driving with an ac magnetic field (setup shown in Figure $2 b$ ).

To demonstrate the working principle of this ME conversion in a wide range of frequencies and to characterize the output voltage generated via stress, an ac magnetic field with frequency $0.5-5 \mathrm{~Hz}$ was produced by the solenoid coil as shown in Figure $2 \mathrm{~b}$ using a current amplifier (Kepco Bipolar Operational Power Supply/Amplifier $\pm 20 \mathrm{~V}, \pm 10 \mathrm{Amp}$ ) driven by a functional generator (Agilent $33220 \mathrm{~A}$ ). We investigated three regimes corresponding to different pre-stress conditions: (i) when the crystal is pre-stressed below the $F_{R}-F_{O}$ transition (linear mode with $F_{R}$ phase); (ii) close to the transition for the stress-induced $\mathrm{F}_{\mathrm{R}}-\mathrm{F}_{\mathrm{O}}$ nonlinear phase transformation (PT) region; and (iii) above critical stress sufficient to bring the crystal to $\mathrm{F}_{\mathrm{O}}$ phase.

As the magnetic field is swept sinusoidally around a bias field, the Galfenol rod strains (see Figure 3a) and stresses the piezocrystal, thus producing a voltage. The voltage generated across the [011] electrodes of the crystal $\left(\mathrm{V}_{\text {crystal }}\right)$ under the three different pre-stress conditions described above is illustrated in Figure $3 b$. Away from the PT region, there is still some voltage produced due to the linear piezoelectric effect. This results in a magnetic field dependent induced voltage due to the large piezoelectric effect in PIN-PMN-PT, regardless of the level of pre-stress applied. When the crystal is in either the $F_{R}$ or $F_{O}$ phases, a slight increase in voltage occurs where the piezomagnetic coefficient $(\mathrm{d} \lambda / \mathrm{d} H)$ of Galfenol is maximum (right axis on Figure 3a), taking into account some mechanical hysteresis due to the fixture as observed in Figure $3 \mathrm{~b}$. However, when the crystal is situated at a critical stress near the phase transition, 
large and abrupt spikes in the voltage of around $50 \mathrm{~V}$ were observed in both the up and down magnetic field sweeps that can only result directly from the stress-induced phase transformation (see Figure 3b). As shown in Figure 1a,b, the hysteresis in the elastic response for $F_{R}-F_{O}$ phase transformation is $\sim 3 \mathrm{MPa}$. This stress variation should be less than that generated by the magnetostrictive strain in the Galfenol rods for an applied magnetic field created by the coil. It should be noted that Gao et al. used similar circuitry with the viability to power low frequency ME magnetic sensors by using Metglas/PMN-PT laminates [15].

The figure-of-merit (FOM) of the magnetic and electric energy conversion in the multi-domain harvester device that represents the efficiency of transduction between magnetic and electric fields is the ME coupling coefficient between the two phases denoted as

$$
\alpha_{M E}=\frac{1}{t} \frac{d V}{d H}
$$

where $V$ is the generated voltage and $t$ is the thickness of the piezoelectric phase. By taking the derivative of the present magnetic field dependent voltage plots and normalizing by the thickness of the crystal, we can obtain the spontaneous values of $\alpha_{M E}$ as a function of bias magnetic field. At $1 \mathrm{~Hz}$ applied field, the maximum ME coefficient was found to be $1.38,1.34$, and $82.9 \mathrm{~V} /(\mathrm{cm}$ Oe) when PIN-PMN-PT is in the $F_{R}$ phase, $F_{O}$ phase, and near the $F_{R}-F_{O} P T$ region, respectively. It is clear that the values of $\alpha_{M E}$ are maximized when the crystal is near the phase transformation due to the large voltage spikes rising from this first-order phase transition and resulting large change in polarization. The value of $\alpha_{M E}$ is an order of magnitude larger as compared to previous results in the literature (see review in Reference [16]).

The potential for storing the harvested energy in an external capacitor was validated by placing the ME harvester in a coil supplying a time varying magnetic field up to $500 \mathrm{G}$. To optimize the response of the Galfenol rods, a magnetic field bias of $250 \mathrm{G}$ was chosen. The time dependent magnetic field and the resulting time dependent current and voltage from the harvester are shown in Figure 3c. For one period of the magnetic field, two voltage spikes corresponding to the $F_{R}-F_{O}$ and $F_{O}-F_{R}$ phase transformations are observed after rectification. An average peak power $\left(\mathrm{P}=\mathrm{V}^{2} / \mathrm{R}\right)$ of $2 \mathrm{~mW}$ was generated in a $1 \mathrm{M} \Omega$ load resistor. This power was then used to charge a $0.47 \mu \mathrm{F}$ capacitor. The circuit diagram is shown in the inset of Figure 4 . The average charging rate was calculated from the slope of the time-dependent voltage over the capacitor $\left(\mathrm{V}_{\text {cap }}\right)$ shown in Figure 4 to be 1.8, 1.6, and $3.2 \mathrm{~V} / \mathrm{s}$ for a $1 \mathrm{~Hz}$ alternating magnetic field when PIN-PMN-PT is in the $F_{R}$ phase, $F_{O}$ phase, and near the $F_{R}-F_{O}$ PT region, respectively. Thus we observe that the increased voltage, magneto-electric coupling and efficiency allow for a 1.8 times increase in charging rate.

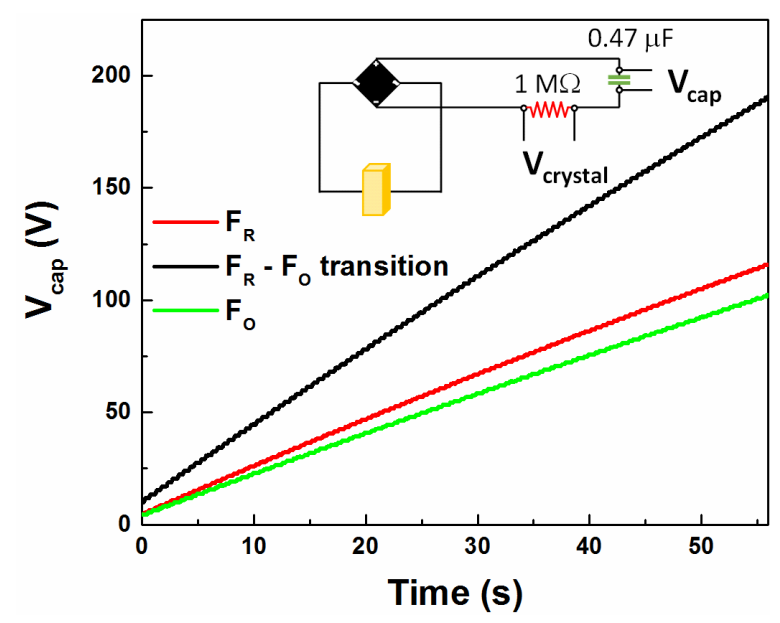

Figure 4. Voltage over a $0.47 \mu \mathrm{F}$ capacitor versus time when the crystal is under various pre-stress conditions. Inset shows circuit diagram used to store voltage from the piezocrystal. 


\section{Demonstration of the Harvester}

As a demonstration of the ME hybrid harvester, a device using $\mathrm{Tb}_{x} \mathrm{Dy}_{1-x} \mathrm{Fe}_{2}$ (Terfenol-D) as the magnetostrictor was constructed. Even though Terfenol-D is expected to have higher strain, it requires larger bias and driving fields. This device was driven by a rotating permanent magnet as shown in Figure 5a [17]. The peak voltage as a function of time is shown on Figure 5b for several regimes corresponding to various magnitude of pre-stress conditions of the single crystal: below PT $\left(\mathrm{F}_{\mathrm{R}}\right)$, through PT, and above PT ( $\mathrm{F}_{\mathrm{O}}$ phase). The voltage peak (Figure $5 \mathrm{~b}$ ) generated across the single crystal was measured using a high impedance electrometer (Keithly 617) and the voltage bias corresponds to different starting charge for various pre-stress bias regimes. An increase in voltage of three times as compared to the $F_{R}$ phase and nine times that of the $F_{O}$ phase is observed at the PT region. It is of relevance to point out that the same peak voltage was maintained as the magnetic field was repeatedly cycled. In previous work, we demonstrated that this $F_{R}-F_{O}$ phase transformation can be cycled for over 40 million times without fatigue or aging effects which increases the suitability of utilizing this phase transformation for harvesting devices [18].

(a)
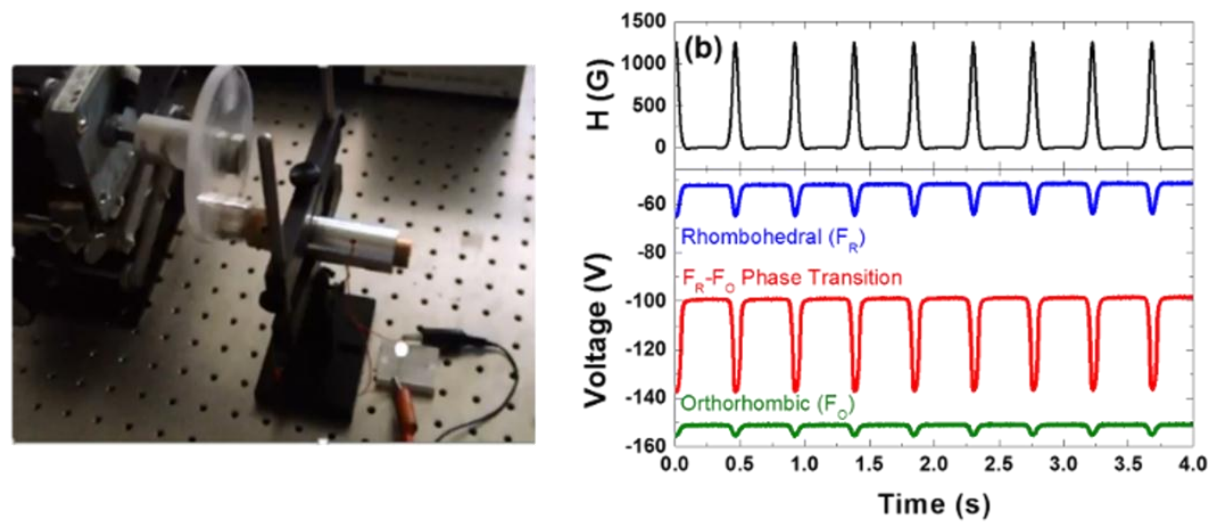

Figure 5. (a) Prototype energy harvester design (patent pending) [17] using Terfenol-D as the magnetostrictor (see video in the Supplementary Files) and (b) voltage response measured over a $22 \mathrm{nF}$ capacitor generated by rotating magnets.

The prototype in Figure 5a demonstrated a larger scale autonomous magnetoelectric energy harvesting device utilizing stray magnetic fields. Looking towards miniaturization of a device operating on the same phase transitional principle, there are practical limitations in how thin the crystal can be fabricated. In addition, eliminating eddy currents in Galfenol requires lamination, but machining of laminated rods may present a challenge for rods less than $\sim 1 \mathrm{~mm}$. However, significant impact could be achieved through reduction of the overall size of the fixture without modification of the active elements that would provide the same power but with lower total device volume.

\section{Conclusions}

In summary, we have demonstrated multi-domain, hybrid magnetoelectric energy harvesters capable of converting both magnetic and mechanical motion in a non-resonant broad band regime. Both the magnetostrictive strain and the power produced by the phase transition in the piezocrystal have previously been demonstrated to be relatively independent of frequency. The magnetoelectric composite system consists of a magnetostrictive element and a PIN-PMN-PT domain engineered relaxor ferroelectric single crystal. High energy and power conversion is observed at a stress-induced inter-ferroelectric phase transformation in a mechanically constrained relaxor ferroelectric single crystal. Although thermal energy harvesting was not demonstrated here, it is an inherent property of the domain engineered crystal and is expected to occur, according to our previous harvesters operating on the phase transition transduction principle [7-9]. The harvester described in this work can thus be 
utilized for broadband multi-domain harvesting of magnetic, mechanical, and thermal energy with high power density per cycle.

Acknowledgments: The authors thank the Office of Naval Research (ONR) for financial support for this work. PF would like acknowledge E. Finkel for support in designing the prototype fixture and useful discussion. R.P.M. and J.K. are grateful for the support provided by the Naval Research Enterprise Internship Program (NREIP).

Author Contributions: P.F. conceived the idea and designed the experiments and the harvester, R.P.M, J.K. and M.S. performed testing of the harvester and carried out magnetoelectric experiments and data analysis. M.W.F. and J.R. are responsible for magnetostriction measurements. J.S. performed the piezoelectric material characterization. P.F., M.S. and A.A. wrote the manuscript.

Conflicts of Interest: The authors declare no conflict of interest.

\section{References}

1. Roundy, S.; Wright, P.K. A piezoelectric vibration based generator for wireless electronics. Smart Mater. Struct. 2004, 13, 1131-1142. [CrossRef]

2. Kim, S.-G.; Priya, S.; Kanno, I. Piezoelectric MEMS for energy harvesting. MRS Bull. 2012, 37, $1039-1050$. [CrossRef]

3. Toprak, A.; Tigli, O. Piezoelectric energy harvesting: State-of-the-art and challenges. Appl. Phys. Rev. 2014, 1. [CrossRef]

4. Tang, L.; Yang, Y.; Soh, C.K. Toward broadband vibration-based energy harvesting. J. Intell. Mater. Syst. Struct. 2010, 21, 1867-1897. [CrossRef]

5. Ottman, G.K.; Hofmann, H.F.; Lesieutre, G.A. Optimized piezoelectric energy harvesting circuit using step-down converter in discontinuous conduction mode. IEEE Trans. Power Electron. 2003, 18, 696-703. [CrossRef]

6. Lefeuvre, E.; Audigier, D.; Richard, C.; Guyomar, D. Buck-boost converter for sensorless power optimization of piezoelectric energy harvester. IEEE Trans. Power Electron. 2007, 22, 2018-2025. [CrossRef]

7. Dong, W.D.; Finkel, P.; Amin, A.; Lynch, C.S. Giant electro-mechanical energy conversion in [011] cut ferroelectric single crystals. Appl. Phys. Lett. 2012, 100. [CrossRef]

8. Moyet, P.R.; Stace, J.; Amin, A.; Finkel, P.; Rossetti, G.A., Jr. Non-resonant electromechanical energy harvesting using inter-ferroelectric phase transitions. Appl. Phys. Lett. 2015, 107. [CrossRef]

9. Dong, W.D.; Finkel, P.; Amin, A.; Lynch, C.S. Stress dependence of thermally driven pyroelectric charge release during FER-FEO phase transformations in [011] cut relaxor ferroelectric crystals. Appl. Phys. Lett. 2012, 100. [CrossRef]

10. Finkel, P.; Benjamin, K.; Amin, A. Large strain transduction utilizing phase transition in relaxor-ferroelectric $\mathrm{Pb}\left(\mathrm{In}_{1 / 2} \mathrm{Nb}_{1 / 2}\right) \mathrm{O}_{3}-\mathrm{Pb}\left(\mathrm{Mg}_{1 / 3} \mathrm{Nb}_{2 / 3}\right) \mathrm{O}_{3}-\mathrm{PbTiO}_{3}$ single crystals. Appl. Phys. Lett. 2011, 98. [CrossRef]

11. Finkel, P.; Amin, A.H.; Benjamin, K.C. Crystalline relaxor-ferroelectric phase transition transducer. US Patent 8,604,676 B1, 2013.

12. Heitmann, A.A.; Rossetti, G.A., Jr. Thermodynamics of ferroelectrics solid solution with morphotropic phase boundaries. J. Am. Ceram. Soc. 2014, 97, 1661-1685. [CrossRef]

13. Clark, A.E.; Wun-Fogle, M.; Restorff, J.B.; Lograsso, T.A.; Schlagel, D.L. Magnetostrictive Galfeno/Alfenol single crystal alloys under large compressive stresses. In Proceedings of the 7th International Conference on New Actuators, Bremen, Germany, 19-21 June 2000; pp. 111-115.

14. Wun-Fogle, M.; Restorff, J.B.; Clark, A.E.; Dreyer, E.; Summers, E. Stress annealing of Fe-Ga transduction alloys for operation under tension and compression. J. Appl. Phys. 2005, 97. [CrossRef]

15. Gao, J.; Wang, Z.; Shen, Y.; Li, M.; Wang, Y.; Finkel, P.; Li, J.; Viehland, D. Self-powered low noise magnetic sensor. Mater. Lett. 2012, 82, 178-180. [CrossRef]

16. Ma, J.; Hu, J.; Li, Z.; Nan, C.W. Recent progress in multiferroic magnetoelectric composites: From bulk to thin films. Adv. Mater. 2011, 23, 1062-1087. [CrossRef] [PubMed]

17. Finkel, P.; Amin, A.H.; Pérez Moyet, R.; Wun-Fogle, M.; Restorff, J.B.; Stace, J.; Murphy, C. Magnetoelectric energy harvesting. US Patent Application Navy Case 102287, 2014. 
18. Finkel, P.; Amin, A.; Lofland, S.; Yao, J.; Viehland, D. Phase switching at low field and large sustainable strain output in domain engineered ferroic crystals. Phys. Status Solidi A 2012, 209, 2108-2113. [CrossRef]

(C) 2015 by the authors; licensee MDPI, Basel, Switzerland. This article is an open access article distributed under the terms and conditions of the Creative Commons by Attribution (CC-BY) license (http://creativecommons.org/licenses/by/4.0/). 